\title{
Propostas lúdico-pedagógicas nas atividades do Programa Saúde na Escola
}

\author{
Eliabe Rodrigues de Medeiros \\ Bacharel em Enfermagem pela Universidade Federal do Rio Grande do Norte (UFRN) \\ Mestre em Enfermagem pela Universidade Federal do Rio Grande do Norte (UFRN) \\ Especialista em Gestão de Programas e Projetos de Esporte e de Lazer na Escola pelo \\ Instituto Federal de Educação, Ciência e Tecnologia do Rio Grande do Norte (IFRN) \\ Doutorando em Enfermagem pelo Programa de Pós-graduação em Enfermagem \\ da Universidade Federal do Rio Grande do Norte (UFRN) \\ $\triangle$ eliabe.medeiros@hotmail.com
}

Kadydja Karla Nascimento Chagas

Licenciada em Educação Física pela Universidade Federal do Rio Grande do Norte (UFRN) Especialista em Fisiologia do Exercício pela Universidade Veiga de Almeida (UVA) Mestra em Educação pela Universidade Federal do Rio Grande do Norte (UFRN) Doutora em Educação pela Universidade Federal do Rio Grande do Norte (UFRN) Docente do Instituto Federal de Educação, Ciência e Tecnologia do Rio Grande do Norte (IFRN). Natal, RN, Brasil.

\begin{abstract}
Resumo:
O Programa Saúde na Escola oferta ações de saúde para a rede pública de ensino, mas elas são executadas, principalmente, por meio de palestras e aulas expositivas que podem comprometer a efetividade dos seus objetivos. As práticas lúdico-pedagógicas são estratégias que podem contribuir com a geração de resultados satisfatórios. Objetivou-se identificar as atividades propostas pelo Programa Saúde na Escola e analisar se apresentam características lúdico-pedagógicas. Foi realizada uma pesquisa documental com de materiais publicados nos portais dos Ministérios da Saúde e da Educação. Seis documentos foram identificados e 162 atividades extraídas. A análise da presença das características lúdico-pedagógicas considerou os critérios: social, cultural, educacional, imaginária, reguladora, livre e espontânea, temporal e espacial, e diversão e o prazer. $38,27 \%$ das atividades foram publicadas em 2013 , 88,3\% orientam atividades de caráter preventivo e de promoção da saúde, 17,3\% abordam a temática da alimentação e nutrição, $13,0 \%$ a cultura de paz e $13,0 \%$ a saúde indígena. Identificou-se ainda que $68,5 \%$ das atividades apresentaram características lúdico-pedagógicas e foram mais frequentes nas temáticas de cultura de paz $(11,2 \%)$, saúde indígena $(10,5 \%)$, práticas corporais, atividade física e lazer $(10,5 \%)$ e sobre educação em direitos humanos (9,9\%). Em sua maioria, as atividades do Programa Saúde na Escola foram publicadas em 2013, integram o componente II do programa e se destinam a trabalhar a temática de alimentação e nutrição. As características lúdico-pedagógicas estão presentes na maior parte das atividades e mostra-se relevante para o aprendizado das temáticas, mudança de hábitos e melhoria da qualidade de vida.

Palavras-chave: Saúde, Educação, Ensino, Jogos e brinquedos, Serviços de saúde escolar.
\end{abstract}

\section{Playful pedagogical proposals in the activities of the Programa Saúde na Escola (School Health Program)}

\begin{abstract}
:
The Programa Saúde na Escola (School Health Program) offers health actions for the public school system, but they are mainly implemented through lectures and lectures that can compromise the effectiveness of
\end{abstract}


its objectives. Playful-pedagogical practices are strategies that can contribute to the generation of satisfactory results. The objective was to identify the activities proposed by the Health at School Program and to analyze whether they present playful-pedagogical characteristics. A documentary research was carried out with materials published on the portals of the Ministries of Health and Education. Six documents were identified and 162 activities extracted. The analysis of the presence of playful-pedagogical characteristics considered the criteria: social, cultural, educational, imaginary, regulatory, free and spontaneous, temporal and spatial, and fun and pleasure. 38.27\% of the activities were published in $2013,88.3 \%$ guide preventive and health promotion activities, $17.3 \%$ address the theme of food and nutrition, $13.0 \%$ the culture of peace and $13.0 \%$ indigenous health. It was also identified that $68.5 \%$ of the activities presented ludic-pedagogical characteristics and were more frequent in the themes of culture of peace (11.2\%), indigenous health (10.5\%), body practices, physical activity and leisure (10.5\%) and on human rights education (9.9\%). Most of the activities in the Programa Saúde na Escola were published in 2013, are part of component II of the program and are designed to work on the theme of food and nutrition. The playful-pedagogical characteristics are present in most activities and are relevant for learning the themes, changing habits and improving the quality of life.

Keywords: Health, Education, Teaching, Play and playthings, School health services.

\section{Propuestas lúdico-pedagógicas en las actividades del Programa Saúde na Escola (Programa Salud en la Escuela)}

\section{Resumen:}

El Programa Saúde na Escola (Programa Salud en la Escuela) ofrece acciones de salud para el sistema escolar público, pero se implementan principalmente a través de charlas y charlas que pueden comprometer la efectividad de sus objetivos. Las prácticas lúdico-pedagógicas son estrategias que pueden contribuir a la generación de resultados satisfactorios. El objetivo fue identificar las actividades propuestas por el Programa Salud en la Escuela y analizar si presentan características lúdico-pedagógicas. Se realizó una investigación documental con materiales publicados en los portales de los Ministerios de Salud y Educación. Se identificaron seis documentos y se extrajeron 162 actividades. El análisis de la presencia de características lúdico-pedagógicas consideró los criterios: social, cultural, educativo, imaginario, normativo, libre y espontáneo, temporal y espacial, y diversión y placer. $38,27 \%$ de las actividades fueron publicadas en 2013 , el $88,3 \%$ orientan actividades preventivas y de promoción de la salud, el 17,3\% abordan el tema de alimentación y nutrición, el 13,0\% la cultura de paz y el 13,0\% \% salud indígena. También se identificó que el 68,5\% de las actividades presentaban características lúdico-pedagógicas y eran más frecuentes en los temas de cultura de paz $(11,2 \%)$, salud indígena $(10,5 \%)$, prácticas corporales, actividad física y ocio $(10,5 \%)$ y educación en derechos humanos (9,9\%). La mayoría de las actividades del Programa Saúde na Escola se publicaron en 2013, forman parte del componente II del programa y están diseñadas para trabajar en el tema de alimentación y nutrición. Las características lúdico-pedagógicas están presentes en la mayoría de actividades y son relevantes para aprender los temas, cambiar hábitos y mejorar la calidad de vida. Palabras clave: Salud, Educación, Enseñanza, Juego e implementos de juego, Servicios de salud escolar.

\section{INTRODUÇÃO}

O Programa Saúde na Escola é uma política pública brasileira que oferta ações de detecção de doenças e prevenção de agravos, promoção e atenção às necessidades de saúde em educandos. Suas atividades estão propostas para serem executadas em creches, pré- 
escolas, ensino fundamental e médio, educação tecnológica e profissionalizante e Educação de Jovens e Adultos (EJA) (MEDEIROS e PINTO, 2018).

As ações do programa devem ser executadas em caráter contínuo e são organizadas em três componentes: avaliação clínica e psicossocial (Componente I), promoção da saúde e prevenção de agravos (Componente II) e formação dos profissionais e educação permanente (Componente III) (LOPES; NOGUEIRA; ROCHA, 2018; MACHADO et al., 2015). Essas atividades deverão englobar diversas temáticas no sentido de responder às demandas da comunidade escolar (BRASIL, 2013a; SILVA et al., 2017).

Ao início de cada período letivo é proposto pela gestão do programa que uma semana de mobilização seja realizada nas escolas a fim de que a comunidade escolar conheça e se envolva nas atividades do programa com enfoque em uma temática prioritária definida de acordo com as necessidades de saúde (BRASIL, 2015a).

Como meio de subsidiar que isso aconteça os Ministérios da Saúde e da Educação brasileiros dispõem de relatos de experiência, manuais temáticos e guias de atividades orientadoras para auxiliar na realização dessas atividades. Tais documentos contêm informações sobre ações de diversas temáticas que podem ser realizadas por profissionais de saúde e educação, além do público-alvo, recursos materiais, tempo e modo de execução, entre outras características.

Apesar disso, o que se observa é a execução das atividades do Programa Saúde na Escola mais voltada a oferta de palestras e aulas expositivas (SOUSA; ESPERIDIÃO; MEDINA, 2017). Esse formato apresenta características, a exemplo da explanação de informações que devem ser memorizadas pelos alunos, ocasião que não é possível identificar em uma atividade considerada lúdica.

Nesses casos, o profissional de saúde vai até a escola para realizar uma ação de saúde sobre determinada temática em dia que lhe é adequado ou possível. A proposta de planejamento e execução da ação juntamente com o professor acaba sendo comprometida, visto que não há momento de diálogo prévio e troca de conhecimentos de saúde e pedagógico entre esses profissionais. Situações como essa podem comprometer o objetivo do programa de contribuir para a promoção do desenvolvimento saudável dos educandos, o qual deve acontecer mediante a realização de atividades efetivas (MASSA, 2015). 
Entende-se que a articulação dos profissionais de saúde e de educação podem contribuir para a realização de atividades que tragam resultados satisfatórios para os estudantes e isso pode acontecer por intermédio das práticas lúdico-pedagógicas.

O processo de ensino-aprendizagem das condições saudáveis mediado por essas práticas se dá com critérios que incentivam o desenvolvimento de uma aprendizagem afetiva ao promover a curiosidade sobre determinado assunto. Também contribui com a interação entre os participantes através de situações de reciprocidade e intencionalidade (COSCRATO; PINA; MELLO, 2010).

As práticas lúdico-pedagógicas podem ser executadas mediante o uso de jogos, brinquedos e brincadeiras, meio pelo qual é possível incentivar que o estudante adquira o conhecimento sobre determinado aspecto e vivencie o divertimento proporcionado pela ação de brincar (KISHIMOTO, 2017). É através da experiência do lúdico na educação em saúde que o indivíduo se constitui enquanto ser e pode estimular a aprendizagem da ética e da harmonia entre as pessoas e do autodesenvolvimento.

Ao trazer o conceito de ludicidade para o ensino, Lima (2015) considera que esse processo deixa de ser aleatório ou simplesmente intuitivo e o profissional responsável tem subsídio para potencializar o planejamento das suas propostas. Ela fundamentou-se diferentes autores e identificou oito elementos que permitem definir uma proposta pedagógica como lúdica. São elas:

- Social: promove a interação social mediante o convívio humano;

- Cultural: considera contextos, hábitos, conhecimentos, necessidades, mitos e superstições de uma cultura e época;

- Educacional: tida como estratégia para aprendizagem e contribui para o desenvolvimento de aspectos comportamentais, cognitivos, psicológicos, entre outros;

- Imaginária: contribui com a imaginação de situações, o desenvolvimento de fantasias com a liberdade de ação para errar e recomeçar;

- Reguladora: tem regras explícitas ou implícitas, mesmo não sendo fixas e rígidas, mas que são necessárias para o controle dos participantes;

- Livre e espontânea: deve proporcionar a liberdade de ação mesmo tendo regras preestabelecidas e se preza com a voluntariedade e espontaneidade; 
- Temporal e espacial: determinam um lugar e horário para que a atividade aconteça;

- Diversão e o prazer: carregam consigo a alegria e o divertimento e permitem aos participantes desenvolverem sensação de empoderamento, domínio e autoafirmação.

Diante dessas colocações, surgiu o seguinte questionamento: as atividades propostas pela gestão do Programa Saúde na Escola possuem características que permitem defini-las como lúdico-pedagógicas?

Compreender se o lúdico enquanto prática pedagógica está presente as atividades do Programa Saúde na Escola é de fundamental importância, à medida que tais respostas podem subsidiar que outros estudos sejam realizados na busca pelo aprofundamento das discussões concernentes ao tema. Além disso, os achados desta pesquisa podem auxiliar com que os responsáveis pelo planejamento e execução do Programa Saúde na Escola apropriem-se das práticas lúdicas no intuito de contribuir para o aprimoramento das práticas de saúde na escola e consequente efetividade das atividades de promoção da saúde realizadas neste espaço.

Com isso, o objetivo deste estudo foi identificar as atividades propostas pelo Programa Saúde na Escola e analisar se apresentam características lúdico-pedagógicas.

\section{MATERIAIS E MÉTODOS}

Trata-se de uma pesquisa de abordagem quantitativa do tipo documental, que se caracteriza pela exploração de fontes documentais e análise de materiais que ainda não receberam tratamento analítico e que podem se reelaborados a partir das considerações propostas (GIL, 2008).

No caso do presente estudo, a exploração se deu em documentos oficiais disponíveis nos portais eletrônicos da gestão do Programa Saúde na Escola, a saber Ministérios da Saúde e da Educação. O processo de busca aconteceu no mês de janeiro de 2018 sem a necessidade 
de busca em base de dados além dos portais citados, visto que, os documentos publicados são disponibilizados gratuitamente e não se referem à pesquisas científicas.

A busca dos documentos nos portais aconteceu no mês de janeiro de 2018 e esse processo está apresentado na Figura 1.

Figura 1. Fluxograma da busca e coleta de dados. Natal, RN, Brasil, 2018.

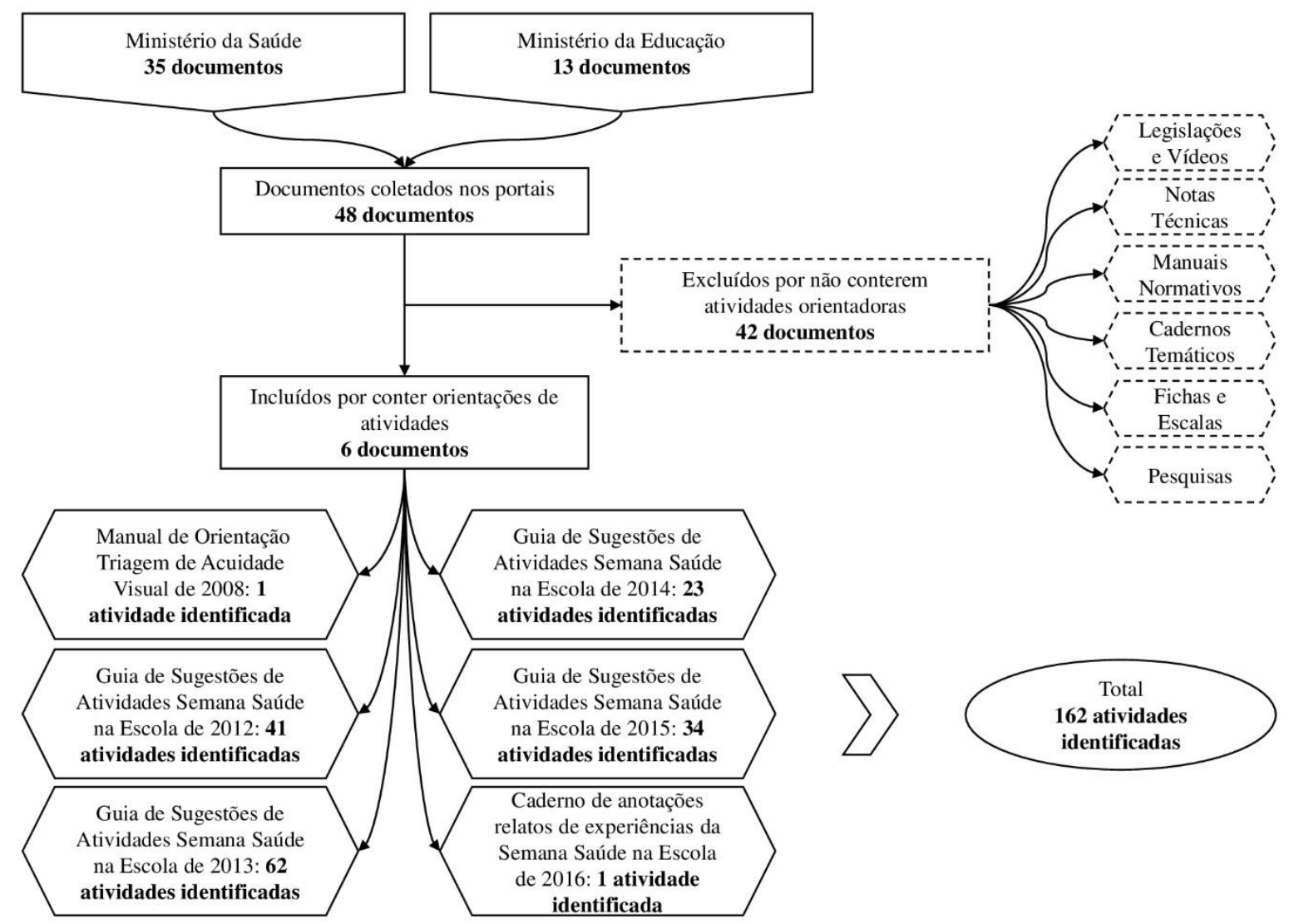

Fonte: autoria própria, 2018.

A busca incluiu todos os documentos relacionados especificamente ao Programa Saúde na Escola e disponibilizados no portal do Ministério da Saúde (35 documentos) e no Ministério da Educação (13 documentos) que totalizaram 48 documentos. Todos esses materiais foram analisados para identificar se contavam com a presença da descrição de atividades propositivas referentes às ações de saúde a serem executadas nas escolas. 
Foram excluídos 42 documentos que não faziam essa descrição e que se tratavam de legislações e vídeos, notas técnicas, manuais normativos, cadernos temáticos, fichas e escalas, como também, pesquisas. Seis documentos (BRASIL, 2008, 2012, 2013b, 2014, 2015b, 2016) continham a descrição de uma ou mais atividades propostas e foram incluídos na análise para identificação e coleta de informações sobre as atividades.

A coleta de dados nos documentos permitiu identificar 162 atividades que foram analisadas de acordo com os critérios para caracterizar uma proposta como lúdica. Além disso, serão utilizadas as variáveis ano de publicação, componente do programa a que se destina e temática proposta.

Os dados foram organizados em planilhas eletrônicas e analisados com base nas dimensões lúdicas de Lima (2015) que nessa pesquisa foram denominadas como características lúdico-pedagógicas. Foi qualificada como atividade lúdico-pedagógica a atividade do Programa Saúde na Escola que apresentou todas as características descritas.

A apresentação dos resultados se deu por meio de figuras contendo gráficos de coluna, pizza e barras, além de tabela com apresentação dos dados em número absolutos e relativos de modo permitir melhor compreensão dos achados.

Por esta pesquisa utilizar como objeto de análise documentos normativos já publicizados e que não contém informações pessoais, não foi necessária apreciação ética por parte de comitê.

\section{RESULTADOS}

A análise das 162 atividades presentes nos seis documentos permitiu compreender que $38,27 \%$ delas foram publicadas no ano de 2013. A maior parte delas (88,3\%) objetivam orientar a execução de atividades de caráter preventivo e de promoção da saúde, já que estão inseridas no Componente II do Programa Saúde na Escola. Além disso, entre as atividades propostas, a maior frequência (17,3\%) tinha o intuito de abordar a temática da alimentação e nutrição, conforme informações apresentadas na Figura 2.

A análise minuciosa das atividades identificadas permitiu conhecer as atividades que apresentaram todas as características lúdico-pedagógicas analisadas. A Tabela 2 apresenta essas atividades de acordo com as temáticas propostas. 
Figura 2. Caracterização das atividades propostas pelo Programa Saúde na Escola em valores percentuais. Natal, RN, Brasil, 2018

\section{Caracterização por ano}

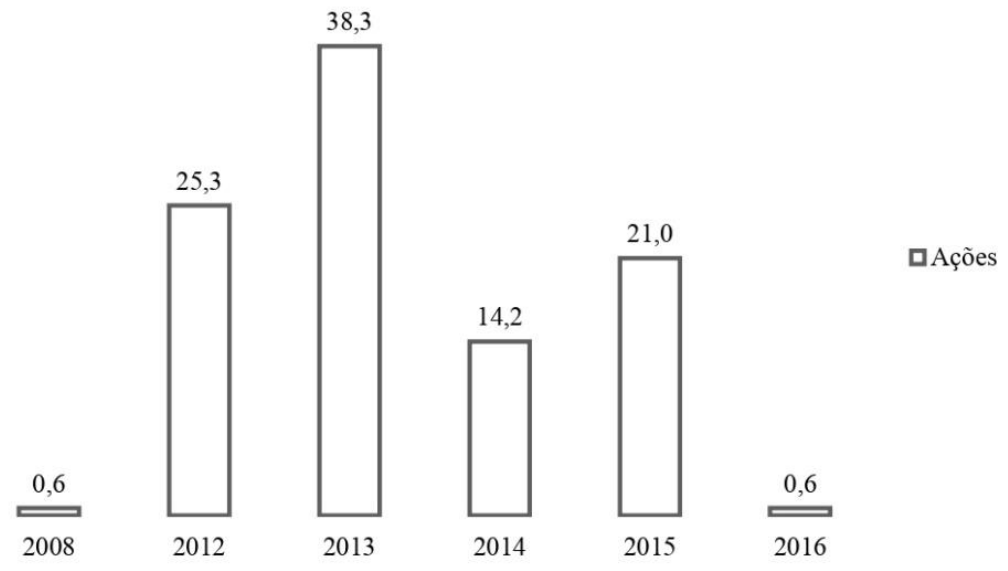

Caracterização por componente no qual se insere

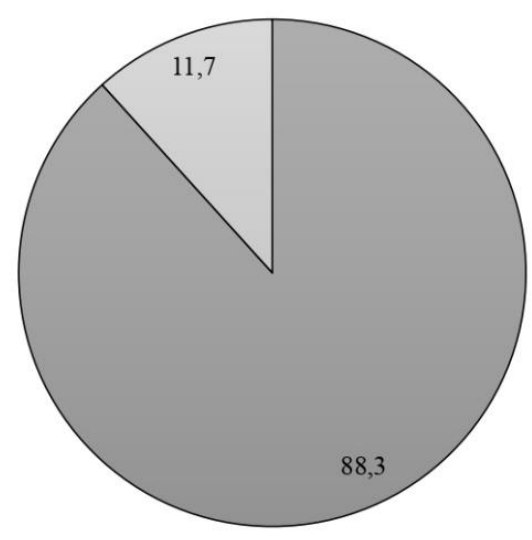

๑Ações do Componente II

$\square$ Ações do Componente I

\section{Caracterização por temática}

Situação vacinal $\square 0,6$

Saúde ambiental $\quad 0,6$

Prevenção ao uso de álcool, tabaco,... 3,0

Saúde ocular $\square 3,7$

Sexualidade e saúde reprodutiva

Educação em direitos humanos

Protagonismo infanto-juvenil
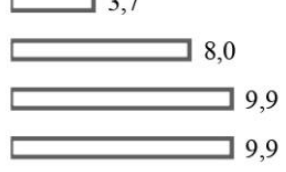

Práticas corporais, atividade fisica e lazer

Práticas intersetoriais

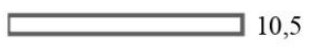

Saúde indígena

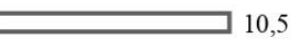

Cultura de paz

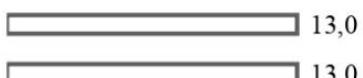

Alimentação e nutrição

Fonte: dados da pesquisa, 2018. 
Tabela 2 - Análise das características lúdico-pedagógicas nas atividades do Programa Saúde na Escola. Natal, RN, Brasil, 2018

\begin{tabular}{|c|c|c|c|c|}
\hline \multirow{3}{*}{ Variável } & \multicolumn{4}{|c|}{$\begin{array}{c}\text { Apresenta características } \\
\text { lúdico-pedagógicas? }\end{array}$} \\
\hline & \multicolumn{2}{|c|}{ Sim } & \multicolumn{2}{|c|}{ Não } \\
\hline & $\mathbf{n}$ & $\%$ & $\mathbf{n}$ & $\%$ \\
\hline Cultura de paz & 18 & 11,2 & 3 & 1,8 \\
\hline Saúde indígena & 17 & 10,5 & 4 & 2,5 \\
\hline Práticas corporais, atividade física e lazer & 17 & 10,5 & - & \\
\hline Educação em direitos humanos & 16 & 9,9 & - & \\
\hline Alimentação e nutrição & 15 & 9,3 & 13 & 8,0 \\
\hline Sexualidade e saúde reprodutiva & 10 & 6,2 & 3 & 1,8 \\
\hline Protagonismo infanto-juvenil & 6 & 3,7 & 10 & 6,2 \\
\hline Práticas intersetoriais & 5 & 3,0 & 12 & 7,5 \\
\hline Saúde ocular & 4 & 2,5 & 2 & 1,2 \\
\hline Prevenção ao uso de álcool, tabaco, crack e outras drogas & 3 & 1,8 & 2 & 1,2 \\
\hline Saúde ambiental & - & - & 1 & 0,6 \\
\hline Situação vacinal & - & - & 1 & 0,6 \\
\hline Total & 111 & 68,5 & 51 & 31,5 \\
\hline
\end{tabular}

Fonte: dados da pesquisa, 2018.

Compreende-se que $68,5 \%$ da amostra apresentou as características lúdicopedagógicas. Também se observou uma concentração maior das propostas lúdicopedagógicas nas atividades das temáticas de promoção da cultura de paz (11,2\%), saúde indígena (10,5\%), práticas corporais (10,5\%), atividade física e lazer, educação em direitos humanos $(9,9 \%)$ e alimentação e nutrição (9,3\%).

As atividades das temáticas de práticas corporais, atividade física e lazer e as da temática dos direitos humanos foram, exclusivamente, consideradas como práticas lúdicopedagógicas. Já as atividades das temáticas de saúde ambiental e de situação vacinal foram as únicas que não apresentaram características lúdico-pedagógicas. 
As atividades da temática de alimentação e nutrição apresentaram frequência semelhante no quantitativo que detinham as características e as que não possuíam.

\section{DISCUSSÃO}

Os achados desse estudo indicam uma maior proposição das atividades do Programa Saúde na Escola nos anos em que essa intervenção passou por aperfeiçoamento e mais voltadas à promoção da saúde e prevenção de agravos, com destaque para a temática de alimentação e nutrição.

As atividades propostas foram prevalentemente identificadas no ano de 2013. Esta situação pode ter sido influenciada pelo processo de reestruturação no qual passou o programa nesse período, quando foi universalizado, e foi possibilitado a todos os municípios do Brasil fazerem a adesão a essa intervenção. Consta desse período a publicação de portaria específica destinada à realização das atividades da Semana de Mobilização Saúde na Escola (VIEIRA; SAPORETTI; BELISÁRIO, 2016). Esse achado pode justificar a publicação dos documentos com as atividades propostas, os quais foram elaborados para orientarem os profissionais sobre diversas atividades na semana de mobilização e ao longo do período letivo.

Observou-se uma predominância de atividades destinadas à promoção da saúde e prevenção de doenças e agravos em detrimento àquelas que se propõem a realizar avaliação clínica das condições de saúde. Em sentido oposto, pesquisa realizada no âmbito da execução das atividades do programa mostrou que atividades de caráter biomédico são prevalentes e que as atividades de promoção da saúde são referidas em quantidade elementar (SOUSA; ESPERIDIÃO; MEDINA, 2017).

Duas considerações podem ser realizadas a partir deste achado. A primeira é que, por mais que seja proposta uma diversidade de atividades referente à promoção da saúde e prevenção de doenças e agravos, elas são executadas em quantidade menor que as de avaliação das condições de saúde. A segunda proposição é que, tendo as atividades de promoção da saúde e prevenção de doenças e agravos realizadas em quantidade elementar, 
as atividades lúdico-pedagógicas podem, em algum, momento não estarem sendo realizadas, já que a maior parte das propostas correspondem às ações com estas características.

Apesar disso, estudo mostrou que executar atividades educativas sobre a temática das parasitoses intestinais junto aos escolares tem contribuído para aumentar o conhecimento e a, consequentemente, prevenção do contágio. Contudo, é notório que essas ações precisam ser constantes para se alcançar os resultados esperados (BRAGAGNOLLO et al., 2018).

Ressalta-se ainda que, por mais que já estejam definidas nos documentos normativos, a execução das propostas do Programa Saúde na Escola necessita da participação dos profissionais dos setores saúde e educação de forma conjunta. Isso é necessário para que se tenham atividades que foram planejadas considerando o conhecimento dos profissionais da saúde e dos aspectos pedagógicos que são dominados pelos professores (MEDEIROS et al., 2019). A complementação de saberes nesses casos incentiva a efetividade das ações.

Além disso, o programa também preconiza em suas normatizações que estes profissionais sejam capacitados constantemente para tal (BRASIL, 2015) e isso também tem sido identificado como uma das necessidades dos profissionais que atuam no contexto da saúde escolar (MEDEIROS et al., 2018). Por isso, acredita-se que estas capacitações contribuam com a realização destas atividades, visto que possibilita um maior contato dos professores junto aos profissionais de saúde e, consequentemente, sua integração, mediante o compartilhamento de conhecimento próprios de suas formações e enriquecimento das atividades planejadas e executadas.

A predominância de propostas com características lúdico-pedagógicas sugere que as atividades do programa podem se mostrar efetivas quando planejadas e executadas de maneira a priorizar que essas características façam parte dela. Em vista disso, a implementação das atividades com essas características também perpassa pelo que foi afirmado por Penso et al (2013) quando devem atender às particularidades do período de vida dos grupos nas quais são direcionadas e que, é por meio destas propostas lúdicas que é possível adequá-las para as necessidades identificadas no período de desenvolvimento dos estudantes.

Atividades com características lúdico-pedagógicas são de suma importância na execução de ações de educação em saúde, conforme observado no estudo que identificou 
evidências na literatura de que podem contribuir não apenas para o conhecimento sobre determinados temas como também mudar comportamentos e a consequente melhoria da qualidade de vida (COSCRATO; PINA; MELLO, 2010). Somado a isso, a utilização de atividades lúdicas tem oportunizado com que haja uma maior interação do professor junto ao discente, ou profissional de saúde e aluno mostrando-se relevante para o aprendizado das diversas temáticas trabalhadas com o estudante (BOMFIM et al., 2015).

As atividades voltadas a trabalhar a cultura de paz foram as mais frequentes entre as que possuíram características lúdico-pedagógicas. Isso merece destaque diante do cenário atual em que os valores de convivência social estão sendo desrespeitados, comprometem as relações humanas e influenciam diretamente o convívio das pessoas no espaço escolar (NASCIMENTO e SALLES FILHO, 2013). Frente a isso, as atividades dessa temática convergem para a valorização da cidadania e prevenção das violências.

Apesar disso, estudo realizado em uma capital do sudeste do Brasil identificou que nenhuma das escolas avaliadas executaram as atividades relacionadas a temática cultura de paz/combate à violência (OLIVEIRA et al., 2018). Tal achado permite compreender que apesar de ser mais frequente, as ações de saúde escolar voltadas a esta temática, por mais que necessárias, ainda não são difundidas entre os profissionais.

Merece destaque também o fato de as atividades relacionadas à alimentação e nutrição terem apresentado uma distribuição igual entre as que possuem características lúdicopedagógicas e as que não possuem. Isso é explicado pelo fato de muitos escolares apresentarem excesso de peso e ser necessário abordar essa realidade mediante ações de avaliação antropométrica e a explanação de temas relacionados a prática de atividade física, alimentação saudável e nutrição (BATISTA; MONDINI; JAIME, 2017).

Todas essas atividades são propostas e devem ser executadas de modo a dar resposta as diversas necessidades de saúde que a comunidade escolar apresenta. Isso é possível mediante a participação dos diversos atores envolvidos nesses tipos de instituições de modo a oferecer um modelo de cuidado integral a saúde (MÉNDEZ; BARRERA; OSPINA, 2014).

Ressalta-se como limitação do presente estudo o fato de ter sido realizado a partir de documentos normativos. Isso impossibilita a compreensão dos fatores que podem influenciar a aplicabilidade prática dessas ações. 


\section{CONCLUSÃO}

Foi identificado que as atividades propostas pelo Programa Saúde na Escola foram, em sua maioria, divulgadas no ano de 2013 quando a intervenção foi universalizada com a possibilidade de adesão para todos os municípios do país. Elas integram, principalmente, o Componente II, característica que ressalta a importância do programa na promoção ao desenvolvimento de hábitos saudáveis. Isso pôde ser observado em ações de diferentes temáticas, com destaque para aquelas que trabalham as temáticas de alimentação e nutrição.

A análise dessas atividades indicou que a maior parte delas contém características lúdico-pedagógicas e que a frequência maior se refere as temáticas de cultura de paz, saúde indígena e práticas corporais, atividade física e lazer. Atividades com essas características são importantes estratégias para promover a interação entre os profissionais de saúde, educação e educandos, principal público a que se destina. Também contribui para o aprendizado da temática trabalhada, mudança de hábitos e melhoria da qualidade de vida.

Diante disso, espera-se que os achados deste estudo possam contribuir para a realização de outras pesquisas, como é o caso de estudos que avaliem a aplicabilidade dessas atividades com características lúdico-pedagógicas e suas efetividades. Almeja-se também que profissionais e gestores envolvidos no Programa Saúde na Escola utilizem esse estudo no sentido de perceber a importância das propostas lúdico-pedagógicas já oferecidas em manuais do referido programa.

\section{REFERÊNCIAS}

BOMFIM, A. M. A.; SOUZA, M. E. C. A.; ROCHA, M. C G.; PORTO, V. F A.; LIMA, E. B.; MESQUITA, T. M. Recurso lúdico no processo de educação em saúde em crianças de escolas públicas de Alagoas: relato de experiência. Interfaces - Revista de Extensão da UFMG, Belo Horizonte, v. 3, n. 1, p. 117-121, 2015. Disponível em: <https://www.ufmg.br/proex/revistainterfaces/index.php/IREXT/article/view/108/pdf>. Acesso em: 18 set. 2019.

BATISTA, M. S. A.; MONDINI, L.; JAIME, P. C. Ações do Programa Saúde na Escola e da alimentação escolar na prevenção do excesso de peso infantil: experiência no município de Itapevi, São Paulo, Brasil, 2014. Epidemiologia e Serviços de Saúde, Brasília, v. 26, n. 3, p. 569-578, 2017. Disponível em: <https://www.scielo.br/pdf/ress/v26n3/2237-9622-ress-26-03-00569.pdf>. Acesso em: 11 dez. 2020.

BRAGAGNOLLO, G. R.; GODOY, P. C. G. T.; SANTOS, T. S.; RIBEIRO, V. S.; MORERO, J. A. P.; FERREIRA, B. R. Intervenção educacional sobre enteroparasitoses: um estudo quase experimental. Revista CUIDARTE, 
Bucaramanga, v. 9 , n. 1, p. 2030-2044, 2018. Disponível em: <https://revistacuidarte.udes.edu.co/index.php/cuidarte/article/view/486/919>. Acesso em: 11 dez. 2020.

BRASIL. Ministério da Saúde e Ministério da Educação. Triagem de Acuidade Visual: Manual de Orientação. Brasília: Ministério da Saúde, 2008.

. Ministério da Saúde e Ministério da Educação. Semana Saúde na Escola: Guia de Sugestões de Atividades. Brasília: Ministério da Saúde, 2012.

. Ministério da Saúde e Ministério da Educação. Manual Instrutivo: Programa Saúde na Escola. Brasília: Ministério da Saúde, 2013a.

Ministério da Saúde. Secretaria de Atenção à Saúde. Departamento de Atenção Básica. Guia de Sugestões de Atividades: Semana Saúde na Escola. Brasília: Ministério da Saúde, $2013 \mathrm{~b}$.

. Ministério da Saúde e Ministério da Educação. Guia de Sugestões de Atividades: Semana Saúde na Escola. Brasília: Ministério da Saúde, 2014. 2015a.

. Ministério da Saúde. Ministério da Educação. Caderno do Gestor do PSE. Brasília: Ministério da Saúde,

Ministério da Saúde e Ministério da Educação. Semana Saúde na Escola: Guia de Sugestões de Atividades. Brasília: Ministério da Saúde, 2015b.

. Ministério da Saúde. Secretaria de Atenção à Saúde. Departamento de Atenção Básica. Caderno de Anotações: Relatos de Experiências da Semana Saúde na Escola. Brasília: Ministério da Saúde, 2016.

COSCRATO, G.; PINA, J. C.; MELLO, D. F. Use of recreational activities in health education: Integrative review of literature. Acta Paulista de Enfermagem, São Paulo, v. 23, n. 2, p. 257-263, 2010. Disponível em: <https://www.scielo.br/pdf/ape/v23n2/17.pdf>. Acesso em: 11 dez. 2020.

GIL, A. C. Métodos e técnicas de pesquisa social. $8^{a}$ ed. São Paulo: Atlas, 2008.

KISHIMOTO, T. M. Jogo, Brinquedo, Brincadeira e a Educação. 14ª ed. São Paulo: Cortez, 2017.

LIMA, E. C. C. Concepção, construção e aplicação de atividades lúdicas por licenciandos da área de ensino de ciências. 2015. 156 f. Dissertação (Mestrado em Ensino, História e Filosofia das Ciências e Matemática) Programa de Pós-graduação em Ensino, História, Filosofia das Ciências e Matemática, Universidade Federal do ABC, Santo André, São Paulo, 2015.

LOPES, I. E.; NOGUEIRA, J. A. D.; ROCHA, D. G. Eixos de ação do Programa Saúde na Escola e Promoção da Saúde: revisão integrativa. Saúde em Debate, Rio de Janeiro, v. 42, n. 118, p. 773-789, 2018. Disponível em: <https://www.scielo.br/pdf/sdeb/v42n118/0103-1104-sdeb-42-118-0773.pdf>. Acesso em: 11 dez. 2020.

MACHADO, M. F. A. S.; GUBERT, F. A.; MEYER, A. P. G. F. V.; SAMPAIO, Y.P. C. C.; DIAS, M. S. A; ALMEIDA, A. M. B.; MORAIS, A. P. P.; SILVA, A. C.; CAMPOS, J. S.; CHAGAS, M. I. O.; CHAVES, E. S. The health school programme: a health promotion strategy in primary care in Brazil. Journal of Human Growth And Development, São Paulo, v. 25, n. 3, p. 307-312, 2015. Disponível em: <http://pepsic.bvsalud.org/pdf/rbcdh/v25n3/09.pdf >. Acesso em: 11 dez. 2020.

MASSA, M. S. Ludicidade: da Etimologia da Palavra à Complexidade do Conceito. Aprender - Caderno de Filosofia e Psicologia da Educação, Vitória da Conquista, v. 9, n. 15, p. 111-130, 2015. Disponível em: $<$ https://periodicos2.uesb.br/index.php/aprender/article/view/2460/2029>. Acesso em: 11 dez. 2020.

MEDEIROS, E. R.; PINTO, E. S. G.; PAIVA, A. C. S.; NASCIMENTO, C. P. A.; REBOUÇAS, D. G. C.; SILVA, S. Y. B. Facilidades e dificuldades na implantação do Programa Saúde na Escola em um município do nordeste do Brasil. Revista CUIDARTE, Bucaramanga, v. 9, n. 2, p. 2127-2134, 2018. Disponível em: $<$ https://revistacuidarte.udes.edu.co/index.php/cuidarte/article/view/514/960>. Acesso em: 11 dez. 2020. 
MEDEIROS, E. R.; PINTO, E. S. G. Experience and professional training in the School Health Program. Revista da Escola de Enfermagem da USP, São Paulo, v. 52, e03378, 2018. Disponível em: $<$ https://www.scielo.br/pdf/reeusp/v52/1980-220X-reeusp-52-e03378.pdf>. Acesso em: 11 dez. 2020.

MEDEIROS, E. R.; FEIJAO, A. R.; PINTO, E. S. G.; SANTOS, V. E. P. Professional qualification in the School Health Program from the perspective of Complexity Theory. Escola Anna Nery Revista de Enfermagem, Rio de Janeiro, v. 23, n. 3, e20190035, 2019. Disponível em: <https://www.scielo.br/pdf/ean/v23n3/pt_1414-8145-ean23-03-e20190035.pdf>. Acesso em: 11 dez. 2020.

MÉNDEZ, J. D. M.; BARRERA, C. J. G.; OSPINA, J. F. M. Perfil de responsabilidad social empresarial para las entidades promotoras de salud - régimen contributivo de Bucaramanga y su área metropolitana. Revista CUIDARTE, Bucaramanga, v. 5, n. 1, p. 633-643, 2014. Disponível em: $<$ https://revistacuidarte.udes.edu.co/index.php/cuidarte/article/view/101/pdf_6>. Acesso em: 11 dez. 2020.

NASCIMENTO, A.; SALLES FILHO, N. A. A construção da cultura de paz na escola: Pedagogia da convivência em educação. In: Os desafios da escola pública paranaense na perspectiva do professor. Cadernos do PDE. Brasília, $2013 . \quad$ Disponível

em: $<$ http://www.diaadiaeducacao.pr.gov.br/portals/cadernospde/pdebusca/producoes_pde/2013/2013_uepg_pe d_artigo_adriano_do_nascimento.pdf>. Acesso em: $11 \mathrm{dez} .2020$.

OLIVEIRA, F. P. S. L.; VARGAS, A. M. D.; HARTZ, Z; DIAS, S.; FERREIRA, E. F. Percepção de escolares do ensino fundamental sobre o Programa Saúde na Escola: um estudo de caso em Belo Horizonte, Brasil. Ciência \& Saúde Coletiva, Rio de Janeiro, v. 23, n. 9, p. 2891-2898, 2018. Disponível em: $<$ https://www.scielo.br/pdf/csc/v23n9/1413-8123-csc-23-09-2891.pdf>. Acesso em: 11 dez. 2020.

PENSO, M. A.; BRASIL, K. C. T. R.; ARRAIS, A. R.; LORDELLO, S. R. A relação entre saúde e escola: percepções dos profissionais que trabalham com adolescentes na atenção primária à saúde no Distrito Federal. Saúde e Sociedade, São Paulo, v. 22, n. 2, p. 542-453, 2013 DOI: Disponível em: <https://www.scielo.br/pdf/sausoc/v22n2/v22n2a23.pdf>. Acesso em: 11 dez. 2020.

SILVA, U. O. A.; NASCIMENTO, G. L.; SANTOS, I. K.; AZEVEDO, K. P. M.; SANTOS, C. F.; KNACKFUSS, M. I.; MEDEIROS, H. J. Health program in a Brazilian school. Salud Pública de México, Cuernavaca, v. 59, n. 1, p. 2833, 2017. Disponível em: <https://www.scielosp.org/pdf/spm/2017.v59n1/28-33/en>. Acesso em: 11 dez. 2020.

SOUSA, M. C.; ESPERIDIÃO, M. A.; MEDINA, M. G. Intersectorality in the 'Health in Schools' Program: an evaluation of the political-management process and working practices. Ciência \& Saúde Coletiva, Rio de Janeiro, v. 22, n. 6, p. 1781-1790, 2017. Disponível em: <https://www.scielo.br/pdf/csc/v22n6/en_1413-8123-csc22-06-1781.pdf $>$. Acesso em: 11 dez. 2020.

VIEIRA, L. S.; SAPORETTI, G. M.; BELISÁRIO, S. A. Programa Saúde na Escola: marcos jurídicos e institucionais. Revista Médica de Minas Gerais, Belo Horizonte, v. 26, supl. 8, p. S381-S387, 2016. Disponível em: <http://rmmg.org/exportar-pdf/2183/v26s8a72.pdf>. Acesso em: 11 dez. 2020.

\section{(cc) $\mathrm{Br}$}

Este trabalho está licenciado com uma Licença Creative Commons - Atribuição 4.0 Internacional. 